\title{
L'animalità nella poesia di Jolanda Insana
}

\section{The Animality in the Poetry of Jolanda Insana}

\author{
Paulina Malicka \\ Università Adam Mickiewicz di Poznań \\ pmalicka@amu.edu.pl
}

\begin{abstract}
The scope of the article is to reflect upon the argument of animality in the work of the Sicilian poet Jolanda Insana (1937-2016) which represents one of the possible interpretive keys of her works. The analytical part of the paper contains an analysis from the anti-anthropocentric perspective of some fragments of the section Bestia clandestina included in the volume Turbativa d'incanto (2012).
\end{abstract}

Keywords: animal, sound, voice, trace, territory

Lo scopo del presente contributo è quello di riflettere sul concetto dell'animalità nella scrittura di Jolanda Insana la quale, pur rappresentando una delle voci più originali e temerarie nel panorama poetico italiano, continua inspiegabilmente a porsi ai margini del canone letterario nazionale. La poetessa, nata a Messina nel 1937 e spentasi a Roma nel 2016, è stata scoperta nel 1977 da Giovanni Raboni che definisce la sua prima raccolta tardiva intitolata Sciarra amara nei termini di una lunga "maturazione semiclandestina" (Raboni, 1987, 589) venata dall'esperienza della traduzione dei testi antichi, greci e latini e percepita come un esercizio sulle possibilità estreme del linguaggio. L'intera produzione poetica della messinese abbraccia i testi scritti tra il 1977 e 2012 (Sciarra amara, Fendenti fonici, Il collettame, La clausura, Medicina carnale, L'occhio dormiente, La stortura, La tagliola del disamore) raccolti in un unico libro Tutte le poesie del 2007 nella collana Gli Elefanti Poesia della Garzanti, poi segue Satura di cartuscelle, Turbativa d'incanto e Frammenti di un oratorio per il centenario del terremoto di Messina confluiti nel volume uscito postumo nel 2017 Cronologia delle lesioni. 2008-2013. 
La peculiarità della sua poesia sta nell'uso di un linguaggio ibrido che impasta elementi aulici di tradizione classica e voci appartenenti a registri bassi che sfociano spesso in espressioni vilipendiose e volgari. Le sue sono "parole in guerra", come dirà Maria Antonietta Grignani (Grignani, 2008), superstiti di una "forzatura espressionistica" (Alfano, 2009, 146) e di una "deformazione linguistica" (Donati, 2007, 591). Già nella stessa scelta del titolo della prima raccolta Sciarra amara che allude in siciliano ad un litigio, ad una violenta rissa o zuffa verbale, si delineano $\mathrm{i}$ tratti caratteristici ed inconfondibili dell'espressione poetica della messinese.

La sua è una poesia teatrale, corale, dialogica che va soprattutto ascoltata e non letta con gli occhi, giusto per sentire quel brivido della parola ed i suoi "fendenti fonici" (Insana, 2007, 124) che tagliano come spade e colpiscono "come un colpo d'aria affilata" (Fusini, 2007, 583) facendo travolgere l'ascoltatore da un delirio di suoni aspri, di parole spesso incomprensibili, di quelle "scannaparole" (Insana, 2007, 166), di quei "gabbalessemi" (Insana, 2007, 166), "lustrastilemi" (Insana, 2007, 70) o "sputafonemi" (Insana, 2007, 73), come dice Insana. Il suo stile è segnato dall'uso di vocaboli spesso inventati, suffissati o prefissati, intriso di neologismi, di raddoppiamenti dei sostantivi, verbi, aggettivi ("sbraitar", Insana, 2007, 345; "sdimenticare", "sdesolata", "scoscienziata", Insana, 2007, 46; "spubblicare", Insana, 2007, 46), nonché di numerosi colloquialismi e dialettalismi spesso impastati con l'italiano medio. La sua poesia nasce nel solco tra il sacro e il profano nutrendosi di un linguaggio estremamente violento ("ti cafullo // di moffe e timpolate", Insana, 2007, 32) ed offensivo ("pallidaminchia", Insana, 2007, 48) che sfocia nella perenne bestemmia ("ma chi ti fotte e pensa", Insana, 2007, 19), nella profanazione della parola e nella continua contaminazione di registri. Insana predilige l'ironia, il grottesco, la beffa e l'invettiva, ma nei suoi testi non mancano espressioni colte, grondanti di un sublime lirismo: basti pensare alle ossimoriche "coltellate di bellezza" (Insana, 2007, 143), alla "tagliola del disamore" (Insana, 2007, 449). Insana deforma e spinge il suo linguaggio oltre i limiti del dicibile. Laddove la parola non riesce a reggere la carica espressiva del momento, subentra un termine del tutto escogitato e non si tratta nel suo caso di una esuberante forzatura, ma di una pulsante necessità di comunicare e di dialogare con l'altro non solo per trasmettere un messaggio, ma per farlo vibrare poiché il vero potere della parola è il suo suono. La parola cavia su cui opera chirurgicamente l'autrice viene martoriata ed oltraggiata allo scopo di estrapolarne nuovi sorprendenti significati e recuperarne un nuovo timbro.

Quanto alle scelte formali predilette della lirica insaniana va ricordato un forte influsso della tradizione letteraria con diversi elementi arcaici e popolari: la litania, la filastrocca, il compianto e l'invettiva, nonché una costante presenza della tradizione siciliana con il teatro dei pupi a cui allude il primo componimento della Sciarra amara dal titolo Pupara sono (cf. Lo Castro). I temi che ritornano più spesso nell'opera di Jolanda Insana sono l'esistenza umana percepita appunto come un litigio tra i due lati della stessa identità, la storia dell'isola: i bombardamenti del 
'44, il terremoto di Messina del 1908, l'impegno civile, la cronaca e l'attualità; il femminicidio, l'immigrazione, la guerra, la politica, la malattia, la nutrizione, il cibo, ed infine il mondo vegetale ed animale che offre lo spunto per il nuovo approccio ermeneutico all'opera insaniana.

La nostra riflessione ruoterà, quindi, attorno alla questione dell'animalità, uno dei temi ricorrenti nella poesia della messinese, nonché uno degli argomenti del moderno approccio anti-antropocentrico che toglie l'essere umano dal piedistallo di tutte le forme viventi presenti nel mondo organico. Il nostro studio sulla presenza animale nell'opera poetica di Jolanda Insana è un work in progress che richiede ulteriori approfondimenti ed analisi, sia per quanto riguarda il crescente numero di pubblicazioni ${ }^{1}$ sull'attività poetica della siciliana, sia per quanto concerne lo sfondo teorico incentrato sulla tematica animale. Per ovvi motivi di spazio e di tempo, ci limitiamo a segnalare soltanto una possibile chiave di lettura della sua poesia incentrata sul concetto dell'animalità, senza pretesa di esaurire l'argomento. La letteratura di riferimento è sterminata ${ }^{2}$, perciò nel presente contributo faremo cenno soltanto ad alcune posizioni filosofiche che, a nostro avviso, svelano una particolare affinità con la voce poetica di Jolanda Insana.

La nostra analisi muove in primis dalla lettura della sessione Bestia clandestina facente parte della raccolta Turbativa d'incanto del 2012. Si tratta di nuovo di una sciarra, di un battibecco dialogato tra due lati della stessa identità, di uno scontro tra il sé e tra l'io consapevole. La bestia clandestina, come spiega la poetessa, è un animale che "tutti noi abbiamo ma lo teniamo a bada" (Insana, 2014), è l'altro che ci abita, è quella parte di noi nascosta con cui parliamo e con cui ci scontriamo in continuazione. "Siamo delle bestie complesse" (Insana, 2014) dice Insana in una delle presentazioni del suo ultimo libro, "bestie" destinate all'isolamento, alla reclusione e al digiuno comunicativo. L'unico interlocutore che rimane all'essere umano è quindi il suo doppio che lo rimprovera, minaccia ed insulta. La scissione dell'io di cui parla Insana è una conferma della sua bipolarità che esplode in questi diverbi accesi e graffianti a non finire: "ritira subito quello che hai detto // ma io non ho detto nulla / sei tu che mi stai sgridando (Insana, 2012, 20); ti sfianco e ti sfiato / bestia rincitrullita / nel circo della vanità (Insana, 2012, 20); mi scuci e ti ricuci / blenorragica e garosa // chissà perché lo chiamano gas esilarante / il protossido

\footnotetext{
${ }^{1}$ Si pensi a Vincenza Di Vita, Pastiches di parole e archivi di cartuscelle analisi di un'autoantologia di Jolanda Insana. Analisi della raccolta poetica Satura di cartuscelle di Jolanda Insana, opera di catalogazaione in versi del trentennio 1977-2007, Anna Mauceri, Il trattamento della voce nella poesia di Jolanda Insana (Medicina carnale, L'occhio dormiente, La stortura).

${ }^{2}$ Basti pensare ai testi di Peter Singer (Liberazione animale), Tom Regan (I diritti animali), Jacques Derrida (L'animale che dunque sono), Gilles Deleuze (Mille piani), Giorgio Agamben (Homo sacer. Il potere sovrano e la nuda vita, L'aperto. L'uomo e l'animale), Roberto Esposito (Terza persona. Politica della vita e filosofia dell'impersonale), Roberto Marchesini (Così parlò il postumano), Felice Cimatti (Filosofia dell'animalità), Leonardo Caffo (Fragile Umanità. Il postumano contemporaneo).
} 
d'azoto usato come anestetico // dovresti saperlo tu / che narcotizzata ti sganasci nel sonno / vedendo paralitici e cani in processione (Insana, 2012, 21); ti scaso / clandestina bestia rincoglionita / e ti accompagno alla ghigliottina // ma non capisci? / mostro i denti / per dire che non mordo (Insana, 2012, 28). Questo sdoppiamento dell'io che parla tra sé e sé lo avvicina ad un essere animale capace, secondo quanto sostengono gli etologi, di comunicare all'interno dello stesso gruppo in una sorta di "dialetto locale" dalle frequenze spesso udibili dall'orecchio umano ma incomprensibili per i rappresentanti di altre specie animali. Il "battibecco tra il sé superficiale e quello profondo" (Grignani, 2012) fa pensare a quanta animalità si celi dietro la comunicazione dell'essere umano che si sottrae al confronto verbale con l'altro rifugiandosi in un mutismo inquietante ed insieme violento. L'animalità che, rimanendo sulla scia del pensiero filosofico di Jacques Derrida (Derrida, 2006) o di Élisabeth De Fontenay (De Fontenay, 1998), va considerata soprattutto in riferimento all'incapacità espressiva dell'animale privo di parola e armato di silenzio. In Insana è l'individuo che perde inesorabilmente la capacità di trasmettere messaggi all'altro da sé, ma nello stesso tempo è in continua ed ossessionante esercitazione dialogica con se stesso sperimentando nuovi timbri e suoni quasi cercasse di inventarsi un proprio idioletto. Dell'importanza dell'aspetto fonico parla la poetessa stessa:

[...] le parole sono prima di tutto suono, e mi affascinano e legano, come le cose nella loro vitalità e terribilità. Suoni voci e click fanno il linguaggio animale, e comunicano emozioni: fame, paura, desiderio, piacere, affetto, gioia, dolore. In principio anche le bestie umane uggiolarono e guairono. Come a dire che le parole escono dal corpo, hanno il loro centro negli organi del corpo e sulla pelle, prima che nel cervello. Poi ci siamo evoluti anche nell'apparato fonatorio e dalla lallazione siamo arrivati all'articolazione delle parole e del discorso... Le parole sono tutta una cosa con la biologia, sono biologiche (Insana, 2009, 8).

C'è quindi in Insana un certo collegamento tra il linguaggio umano e quello animale non umano che oltre ad emettere suoni riescono e trarre melodie anche dal proprio corpo (il fruscio del serpente, lo svolazzare dell'uccello, la flessione delle ali). L'elaborazione della parola poetica avviene attraverso il corpo, nello stesso modo in cui i versi dell'animale possono essere accompagnati dagli effetti acustici prodotti dalle sue arti, ali o pinne.

In più, è interessante osservare in questo frangente che la questione dell'espressività poetica è strettamente legata in Insana all'uso del dialetto siciliano inteso dal filosofo Manlio Sgalambro come "momento animale della lingua. Desiderio di animalità. Smettere di essere uomo. Tornare animale" (Sgalambro, 1999, 5). Infine, la scrittura è animale nella misura in cui pretende di dare voce all'altro, di lasciarsi guidare dall'istinto, dal "duro linguaggio di necessità" (Sgalambro, 1999, 5)

Gianni Turchetta ricollega la tematica culinaria a quella zoologica facendo notare che la poesia di Jolanda Insana 
[...] pullula di animali, che peraltro offrono continue occasioni per esplorare e approfondire l'area semantica e simbolica del mangiare, che diventa, volta a volta, ingoiare, divorare, rosicchiare, spolpare, intonandosi con un bestiario amplissimo, dai pescecani ai maialini, e dai porcospini alle scimmie, con uno spazio privilegiato per gli animali piccolissimi, brulicanti e magari francamente disgustosi: formiche pulci lombrichi zecche gechi zanzare-tigre e persino "topi chiaviconi” (Turchetta, 2007, 602).

L'atto di mangiare compiuto dall'io assume quindi una connotazione animalesca. Ciò che appare molto significativo in questo contesto è il mezzo attraverso il quale avviene il processo di deglutizione, ovvero la bocca - un organo che serve anche a comunicare, ad emettere suoni. "La congiunzione fra il nutrirsi e il parlare" (Turchetta, 2007, 602) risulta così inestricabilmente legata alla questione animale, all'imitazione del comportamento delle bestie, nonché al tentativo di riappropriarsi della loro parola, ma non per parlare al loro nome o al posto loro, bensì come loro. L'animale si costituisce in quanto parola, in quanto mezzo dell'identificazione in cui l'io vuole riconoscersi. E per poterlo fare è costretto a lasciare tracce di sé scrivendo. Così chi scrive diventa un animale autobiografico che non sa cancellare le proprie orme. L'animalità della scrittura poetica di Jolanda Insana sta quindi nell'inseguire le tracce, nello spingere la parola oltre i limiti del dicibile. Scrivere nel suo caso è infine un iniziale privarsi di parole consuete quasi per sentire ciò che avverte ogni animale al quale la parola è stata tolta.

Marilena Renda intravede una certa pertinenza tra la poesia della Insana e le osservazioni di Gilles Deleuze e Felix Guattari riguardanti l'ossessione dell'alimento e dei denti (Renda, 2007, 606). A nostro avviso, vi è ancora un altro tratto pertinente tra la scrittura di Jolanda Insana e il pensiero di Gilles Deleuze. Quello cioè dell'animalità. Un interessante spunto per queste considerazioni viene offerto dall'intervista intitolata L'abecedario fatta a Deleuze da Claire Parnet in cui vengono sollevate tra l'altro le questioni quali il concetto del divenire animale, il rapporto uomo-animale, animale-scrittura, il territorio e la deterritorializzazione. Il filosofo francese biasima il "rapporto umano con l'animale" facendo notare quanto sia "spaventoso il modo in cui le persone parlano ai loro animali" (Deleuze, 2005). Prova ribrezzo per qualsiasi dimostrazione di affetto nei confronti dell'animale non umano: "È odioso, non lo sopporto" (Deleuze, 2005). Il sentimento che gli umani provano verso i "loro" animali è di principio "non umano", l'amore decapita la relazione umana che intercorre tra l'uomo e l'animale. Paradossalmente, solo chi considera l'animale in quanto preda, riesce ad instaurare con esso una relazione umana. Ed è l'esempio del cacciatore riportato da Deleuze: " $i$ cacciatori hanno un rapporto stupefacente con gli animali" (Deleuze, 2005). Il vero rapporto umano con l'animale si fonda sul principio di attività intesa come capacità di reagire, l'operosità o l'esplicazione delle proprie abilità fisiche e pratiche. Ne è un esempio, secondo il filosofo, quello delle zecche o delle pulci. "È vero che sono affascinato da bestie 
come i ragni, le zecche, le pulci, sono importanti come cani e gatti. E anche questi sono rapporti con gli animali. Uno che ha le zecche, le pulci cosa vuol dire? Sono rapporti estremamente attivi con gli animali" (Deleuze, 2005). Un'altra caratteristica che affascina il filosofo è il fatto che gli animali, contrariamente a quanto sosteneva Heidegger, non sono privi di mondo. "Ogni animale ha un mondo" (Deleuze, 2005) in quanto possiede la capacità di rispondere e di reagire allo stimolo. E ancora ciò che colpisce il pensatore francese è l'abilità degli animali di emettere segni e di lasciare tracce, nonché il loro comportamento istintivo di stare in agguato che si addice anche alla condizione di chi scrive.

Se lo scrittore è colui che spinge il linguaggio al limite, limite che separa il linguaggio dall'animalità, dal grido, dal canto, in questo caso allora bisogna dire che sì, lo scrittore è responsabile di fronte agli animali che muoiono, il che vuol dire rispondere degli animali che muoiono. Scrivere non per loro, non si scrive per il proprio gatto o per il proprio cane, ma scrivere al posto degli animali che muoiono, significa portare il linguaggio a questo limite. E non c'è letteratura che non porti il linguaggio e la sintassi al limite che separa l'uomo dall'animale. Bisogna stare su questo stesso limite. Credo. [...] Bisogna sempre essere al limite che separa dall'animalità, ma per l'appunto, in modo da non esserne più separati. Così c'è un'inumanità propria al corpo umano e allo spirito umano, ci sono dei rapporti animali con l'animale (Deleuze, 2005).

L'esercizio di scrittura svela, quindi, una certa similitudine con il rapporto uomo-animale. Si tratta di un'esperienza del limite. La poetessa messinese spinge il linguaggio oltre i limiti del dicibile. Varca la soglia della decenza. Il suo è un linguaggio animale nella misura in cui si lascia plasmare dall'istinto, dalla necessità di essere continuamente depistato. La scrittura è un inseguimento, è lo stare dietro alle parole, è lo stare sulla loro traccia, è un continuo movimento, come quello delle formiche, un continuo spostamento di prospettive. Lo illustrano bene i versi de La bestia clandestina:

[...] per arrivare al cibo

le formiche trovano la strada più breve

seguendo le tracce odorifere degli altri

ma tu sei la formica

che rimasta isolata gira in tondo

sulla propria traccia

sempre più in tondo

sempre più tonta

inseguendo la propria traccia

sempre più forte sempre più sua

sempre più in tondo

finché sfinita s'avvita

e crepa stontita (Insana, 2012, 49). 
La poesia di Jolanda Insana offre infinite possibilità di incontro con il mondo animale e svela un particolare interesse per diverse forme di vita animale e vegetale. Alcune raccolte poetiche della Insana tematizzano il problema dell'animalità, altre sfruttano l'immagine dell'animale allo scopo di chiamare l'altro, di definirlo, offenderlo, oltraggiarlo. Gli animali sono una costante nell'opera insaniana, ma la loro funzione e il loro status cambia nell'arco dell'itinerario creativo. Così, nei primi libri di poesia quali Sciarra amara, Fendenti fonici, Il collettame, La clausura, Medicina carnale, L'occhio dormiente, La stortura, La tagliola del disamore, troveremo diversi epiteti animali ("fetente pescestocco", Insana, 2007, 23), espressioni ingiuriose ("ingravidarmi tutta quanta come vacca", Insana, 2007, 120; "il porco che è lui", Insana, 2007, 122; "non fare il lumacone", Insana, 2007, 148), diverse metafore, detti o proverbi in cui ritornano le presenze animali ("troppi ossi per un solo cane", Insana, 2007, 31; "quando la gallina fa l'ovo", Insana, 2007, 64; "non s'agitano moscerini dove non c'è vino", Insana, 2007, 158).

Il rapporto che la poetessa intrattiene con la sua scrittura presenta ancora un'altra analogia con il pensiero deleuziano. Insana interagisce con la sua lingua poetica, quasi come se fosse un organismo vivente. Tale approccio richiama alla mente il concetto deleuziano del divenire animale inteso come "movimento di scambio e ibridazioni continue tra l'uomo e l'animale, che avviene nei comportamenti quotidiani" (Vignola, 2013, 118). Per "comportamenti" Deleuze intende affetti "attribuibili a tutti i corpi" (Vignola, 2013, 119), "costanti comportamentali, identitarie" (Vignola, 2013, 118) che "determinano la specificità" (Vignola, 2013, 119) dell'essere vivente. Seguendo questo ragionamento il filosofo "situa l'uomo e l'animale in una zona di vicinanza - si tratta di fare corpo con l'animale - tracciando [...] un ponte tra etologia ed etica" (Vignola, 2013, 119).

La poetessa lavora sulla propria espressività lirica ritagliandosi uno spazio tutto suo sulla scena poetica italiana, lontano dallo "sperimentalismo della neoavanguardia" (Zorat, 2009, 215). Costruisce e delimita il proprio territorio, proprio come accade per certi animali. E per Deleuze "costruire un territorio [...] è quasi la nascita dell'arte" (Deleuze, 2005). La questione animale in Deleuze è, quindi, fortemente legata all'idea del territorio, del segno e della traccia e di conseguenza svela una particolare analogia con la scrittura. Scrivere è quindi emettere segni, lasciare tropi, marcare lo spazio. E per Insana "il mondo è fatto di segni; fuori del segno non c'è nulla" (Insana, 2009, 12).

Un interessante accumulo di informazioni riguardanti una particolare sensibilità della poetessa nei confronti del mondo animale, viene offerta dall'intervista di Giancarlo Alfano. Si leggano le testimonianze della poetessa:

Ho sempre letto di tutto, dalla cronaca all'etologia alle vite dei santi, perché ero attratta dai vari linguaggi specialistici, dalle differenze di uso lessicale, dalle parole utilizzate in ciascun sapere o dimensione espressiva. Un interesse, anzi una passione che era guidata 
dal desiderio di mescolare le parole, farle risuonare, spostarle: "sgarrare le parole" avrei detto in Fendenti fonici (Alfano, 2009, 6-7).

Sono stata sempre mossa dalla necessità di sperimentare i linguaggi (Alfano, 2009, 8).

E ancora:

L'uomo è una accumulazione di varie bestie. L'identità è in difficoltà già a partire dalle domande: che bestia sono io? E l'altro che è in me che bestia è? Con quante bestie convivo, se in un solo centimetro di pelle ho milioni di altre bestie? [...].

Dall'efèmera all'uomo, tutti abbiamo una vita. [...] E ci siamo noi, e ci sono gli altri. Si badi bene che questo altro, il vivente-altro è poi quel che definisce per noi lo spazio. È in rapporto all'altro che si costruisce il nostro luogo (Insana, 2009, 9).

Insana è molto esplicita nelle sue dichiarazioni e il suo particolare interesse per il mondo animale è innegabile. Le letture etologiche, a cui la poetessa allude nell'intervista, accompagnate, a quanto pare, da attenti studi biologici, trovano evidenti tracce nel corpo del testo poetico. Ne è la dimostrazione l'impressionante quantità di animali che popolano i suoi versi e che vengono chiamati secondo una precisa nomenclatura zoologica. In Insana dietro la parola animale si celano infiniti organismi viventi, innumerevoli sensibilità e schemi comportamentali. Tale scrupolosità nel chiamare l'altro per nome rende giustizia all'esistenza di numerosissime specie che abitualmente vengono incluse all'interno della stessa categoria quale è quella dell'animale ${ }^{3}$.

L'intero corpus poetico della messinese vanta una lunga lista di mammiferi, vertebrati, rettili, insetti e volatili. Tuttavia, nella tassonomia insaniana il posto decisamente privilegiato lo occupano gli insetti, (soprattutto le formiche) a cui occorrerebbe dedicare un'ulteriore ricerca, nonché i rettili il cui rappresentante più frequente è il geco. Questa simpatia per la specie ripugnante fa pensare di nuovo a Deleuze che negli insetti, soprattutto nei ragni, nelle zecche e nei pulci, intravedeva la massima espressione di animalità intesa come capacità di lasciare segni e di rispondere o reagire a singoli stimoli o sostanze eccitanti.

Nelle prime raccolte la poetessa sembra mantenere "un rapporto animale con l'animale", per dirla con Deleuze. Tale osservazione è dovuta al fatto che molto spesso nei suoi versi l'animale viene strumentalizzato, la sua immagine subisce numerose storpiature e i suoi comportamenti molto spesso denudano l'incapacità fisica o mentale dell'essere umano. Il suo è sempre uno sguardo molto acuto e distaccato sull'essere animale, su come e in che condizioni esso vive e sulle attività che esso svolge. Lo dimostrano meglio i seguenti esempi: "anche le pulci hanno la tosse" (Sciarra amara, in Sciarra amara, 30); "anche il cavallo s'inquarta / quando le

\footnotetext{
${ }^{3}$ Cf. J. Derrida (2006), L'animale che dunque sono, trad. italiana di Massimo Zannini (pp. 30, 31), Milano: Jaca Book Spa.
} 
mosche sono tante" (Servizio d'attesa, in Fendenti fonici, 154); "solo la seppia ha i figli in testa" (E venga un nuovo scaricatore, in Fendenti fonici, 156); "la talpa ha scavato sotto terra" (L'urlo di Abû Nuwàs, in L'occhio dormiente, 311); "in Africa milioni di formiche / spolpano polli e sgrassano maialini / e quando vanno all'attacco dell'uomo che dorme / lo intossicano e svenano" (La vita mette nuove foglie, in La stortura, 373); "ma le vespe fecero nido dentro la magnolia" (Manate di smalto, in La stortura, 375); "formiche amazzoni e sanguinarie / assaltano e saccheggiano / gli altri accampamenti / e deportano la prole" (L'artritica falsità, in La stortura, 407); "ma la mano che non formicola / vuole scriverne il nome / su tutti i muri / per marcare territorio / come fanno i gatti con il piscio" (Questa è terra..., in La tagliola del disamore, 439); "i lupi cantano alla luna e cantano i cuccioli / con le vocine stridule / un modo gentile come un altro / di stare insieme / addestrarsi alla caccia" (Corteggiamenti e altro, in La tagliola del disamore, 502); “finisce l'anno / e le balene grige lasciano l'Alaska / e si mettono in viaggio / per la lunga via del mare / e noi appollaiati a queste seggiole di buona seduta / non ci spostiamo d'un millimetro / per procreare e conservare / e così non andiamo da nessuna parte / e temiamo di tutto" (Viatico, in La tagliola del disamore, 555); "anche la cernia nasce femmina / per diventare maschio e copulare" (La bestia clandestina, in Turbativa d'incanto, Insana, 2012, 45); "il delfino si riconosce allo specchio" (L'idiota sottostante, in Turbativa d'incanto, 68); "per sottrarsi ai predatori / il pesce pagliaccio / con i suoi colori sgargiante aranciata / si confina tra i tentacoli / dell'anemone velenoso / e quando resta vedovo / diventa femmina / e spara uova nell'acqua" (Caràmbola, in Turbativa d'incanto, 100); "emergono dagli abissi oceanici / totani giganti senza occhi / e sono spoglie morte" (Caràmbola, in Turbativa d'incanto, 101).

Soltanto nelle ultime raccolte l'atteggiamento della poetessa nei confronti dell'animale non umano subisce un cambiamento. Nella Turbativa d'incanto troveremo diversi passaggi in cui Insana sembra compatire la sorte dei suoi fratelli minori ["e quando sull'erba vedo il calabrone intirizzito / che ha la schiena rivoltata / lo rigiro e col fiato della bocca lo richiamo / alla vita" (L'urlo di Ab̂̂u Nuwàs, in L'occhio dormiente, 315)], fino a sentirsi lei stessa una bestia: "la bestia sono io" (Lo scoramento, in L'occhio dormiente, 288); "ho vissuto come una bestia" (Il peso nelle mani, in La stortura, 381).

Prova pietà per un calabrone, chiama affettuosamente la tartarughina e la formichina riflettendo sul loro destino e preoccupandosi per la loro sorte ["itterica tartarughina / che si nasconde dietro la vetrina / sotto un melone retato // scappa perché ha paura / o ha paura perché scappa?" (VI, in La tagliola del disamore, 524); "ah la ritrosetta formichina ghiotta / che si tufferebbe affogata / se non fosse sigillata nella sua boccetta" (VII, in La tagliola del disamore, 526)]. Le sta a cuore la vita minacciata delle tartarughe: "come succede alle tartarughine / che ingurgitano sacchetti / flottanti in mare aperto / scambiandoli per meduse luccicanti” (Insana, 2017, 20) 
e parla di delfini chiamandoli "persone non-umane" (Insana, 2017, 22-23). Diventa sempre più empatica e sensibile ai suoni emessi dai volatili paragonandoli spesso alla voce dei poeti:

[...] per fare colpo / l'uccello lira danza flettendo / le penne della coda / e canta canta di tutto / canterino imitatore di lambrette e motoseghe / upupe e picchi / canta il clic della Kodak / i faleci di Catullo e l'esametro di Lucrezio / e vuoi che non trovi una femmina? // non sono disponibile / né ora né mai / il richiamo serve a fare prede // non è detto / c'è un'orca nelle fredde acque canadesi / che rimasta orfana e sola / di più di un anno parla con i leoni marini / ma tu non impari nessun dialetto / non canti nessun canto // invece di minacciare e maledire / ti farebbe bene fingerti capodoglio / che finge d'essere sommergibile / sulla piana del mare / o fingerti elefante che confinato nella riserva / lungo l'autostrada Mombasa-Nairobi / fuoriesce dal chiuso e corre sulle strade del mondo / intonando il rombo dei Tir // voglio restare orfana e sola / non voglio parlare con i leoni / non mi serve nessun'altra lingua / non sono una bestia / e tu taci / non fare versi (L'idiota sottostante, in Turbativa d'incanto, Insana, 2012, 70).

Altrove la poetessa riflette sul destino degli animali sempre più minacciati dall'operato dell'essere umano: "come fanno coi tonni i delfini /sulle rotte del Pacifico / ma scortati e scortati / finiscono nelle reti dei pescatori / allertati dai delfini / che nuotano in superficie / e saltano e giocano / suonando le fanfare della lunga marcia / ai tonni sottostante" (Le foglie del decoro, in Turbativa d'incanto, 15); "anche le galline muoiono di crepacuore / ne sono crepate 230 / un intero gallinaio / per l'assalto di un cane randagio" (Turbativa d'incanto, in La bestia clandestina, 27).

La poetessa ammette la presenza dell'altro che è in noi. Conduce un dialogo graffiante con la bestia che la abita e nello stesso tempo si accorge di essere esposta allo sguardo animale: "E ci siamo noi, e ci sono gli altri. L'altro in noi e l'altro fuori di noi” (Alfano, 2009, 9). Insana accetta che c'è 1'altro, che c'è qualcun altro che ci guarda $^{4}$. E questa esperienza la porta verso una scrittura poetica in cui irrompe il desiderio dell'animalità: "ritorno animale" (Il magico quadrato, in Medicina carnale, 244); "sarò un animale impietoso" (Perché ti arrabbi tanto?, in Il collettame, 189); "come un cane ansimo e non abbaio" (La chiacchiera, in La stortura, 419); "Ho consumato tanto di quel tempo / raspando come un cane alla porta della tana" (Viatico, in La tagliola del disamore, 554).

La poetessa, smonta le parole per avvicinarsi all'altro, per accostarsi al mondo animale, per imparare a tradurlo e a comprendere il linguaggio animale, a decifrare i suoni che gli animali emettono o traggono dai loro corpi. E tutto ciò per approcciarsi all'alterità, ma anche per conoscere se stessa, per riconoscersi anche lei

\footnotetext{
${ }^{4}$ In questi ragionamenti risuona l'eco della frase derridiana suggerita da un incrocio di sguardi scambiati tra il filosofo appen a uscito nudo dalla doccia e la sua gatta che lo osserva: "L'animale ci guarda e noi siamo nudi davanti a lui. E pensare comincia forse proprio qui”. J. Derrida (2006), L'animale che dunque sono, trad. italiana di Massimo Zannini, Milano: Jaca Book Spa, p. 68.
} 
un animale, una bestia, per trovare la propria lingua animale. "L'uomo è una accumulazione di varie bestie. L'identità è in difficoltà già a partire da qui: che bestia sono?" - si interroga Insana e non indugia nella risposta: "io sono un'anguilla / e scivolo / scivolo via" (L'idiota sottostante, in Turbativa d'incanto, 64). La poesia è "un'anguilla", diceva Eugenio Montale. Il suo significato scivola, scivola via. E allora "[...] puoi tu / non crederla sorella?" (Montale, 1984, 262). 


\section{BIBLIOGRAFIA}

Agamben, G. (2002). L'aperto. L'uomo e l'animale. Torino: Bollati Boringhieri.

Agamben, G. (2005). Homo sacer. Il potere sovrano e la nuda vita. Torino: Einaudi.

Alfano, G. (2009). Quella ruga che rende visibili. Giancarlo Alfano intervista Jolanda Insana. In J. Insana, Satura di cartuscelle. Roma: Giulio Perrone Editore.

Caffo, L. (2017). Fragile Umanità. Il postumano contemporaneo. Torino: Einaudi.

Cimatti, F. (2013). Filosofia dell'animalità. Roma-Bari: Laterza.

De Fontenay, E. (1998). Le silence des bêtes: la philosophie à l'épreuve de l'animalité. Paris: Fayard.

Deleuze, G. (2005). Abecedario. Film intervista. In C. Parnet (Ed.), 3 DVD, regia di P.A. Boutang, traduione dal francese di I. Bussoni, F. Del Lucchese, G. Passerone. Roma: Derive Approdi.

Deleuze, G., Guattari, F. (2017). Mille piani. Capitalismo e schizofrenia. Nocera: Orthotes.

Derrida, J. (2006). L'animale che dunque sono, trad. italiana di M. Zannini. Milano: Jaca Book Spa.

Di Vita, V. (2011). Pastiches di parole e archivi di "cartuscelle" analisi di un'auto-antologia di Jolanda Insana. Analisi della raccolta poetica Satura di cartuscelle di Jolanda Insana, opera di catalogazaione in versi del trentennio 1977-2007. In C. Borrelli, E. Candela, A.R. Pupino (Eds.), Memoria della modernità. Archivi ideali e archivi reali (vol. II). Atti del XIII Convegno Internazionale della MOD 7-10 giugno 2011 (pp. 425-433). Pisa: Edizioni ETS.

Donati, A. (1994). In ). In Il Giorno, 6 novembre 1994. In J. Insana, Tutte le poesie (pp. 591-592). Milano: Garzanti.

Esposito, R. (2007). Terza persona. Politica della vita e filosofia dell'impersonale. Torino: Einaudi.

Fusini, N. (1982). In Rinascita, 27 agosto 1982 (p. 19). In J. Insana, Tutte le poesie (pp. 583-585). Milano: Garzanti.

Grignani, M.A. (2012). La seconda di copertina. In J. Insana, Turbativa d'incanto. Milano: Garzanti.

Insana, J. (2002). L'incontro gli incontri. In P. Frassica (Ed.), Salvatore Quasimodo nel vento del mediterraneo, Atti del convegno internazionale (Princeton 6-7 aprile 2001). Novara: Interlinea.

Insana, J. (2007). Tutte le poesie. Milano: Garzanti.

Insana, J. (2009). Satura di cartuscelle. Roma: Giulio Perrone Editore.

Insana, J. (2012). Turbativa d'incanto. Milano: Garzanti.

Insana, J. (2017). Cronologia delle lesioni. 2008-2013. Milano: Luca Sossella editore.

Marchesini, R., Caffo, L. (2014). Così parlò il postumano. Anzio-Lavinio: Nova Logos.

Mauceri, A. (2006). Il trattamento della voce nella poesia di Jolanda Insana (Medicina carnale, L'occhio dormiente, La stortura). Allegoria, 54, 57-70, Anno XVIII, settembre-dicembre 2006. Palermo: G.B. Palumbo \& C. Editore.

Montale, E. (1984). L'anguilla. In E. Montale, La bufera e altro, in Tutte le poesie. Milano: Mondadori Editore.

Raboni, G. (1987). In Poeti del secondo Novecento in Storia della letteratura italiana diretta a Emilio Cecchi e Natalino Sapegno, Il Novecento (vol. II) (p. 241). Milano: Garzanti; ora in La poesia che si fa. Cronaca e storia del Novecento poetico italiano 1959-2004, A. Cortellessa (Ed.) (2005), Milano: Garzanti, p. 238. In J. Insana, Tutte le poesie (pp. 589-590). Milano: Garzanti.

Regan, T. (1990). I diritti animali. Milano: Garzanti.

Renda, M. (2005). In Stilos, 16 agosto 2005 (p. 10). In J. Insana, Tutte le poesie (pp. 606-607). Milano: Garzanti.

Sgalambro, M. (1999). Piccole note in margine a Salvo Basso. In S. Basso, Dui. Catania: Edizioni Prova d'autore. 
Singer, P. (2009). Liberazione animale. Milano: Il Saggiatore.

Turchetta, G. (2003). In V. Spinazzola (Ed.), Tirature '03, il Saggiatore (pp. 21-23). Milano. In J. Insana, Tutte le poesie (pp. 601-603). Milano: Garzanti.

Vignola, P. (2013). Divenire-animale. La teoria degli affetti di Gilles Deleuze tra etica ed etologia. In M. Andreozzi, S. Castignone, A. Massaro (Eds.), Emotività animali. Ricerche e discipline a confronto. Milano: Edizioni Universitarie di Lettere Economia Diritto.

Zorat, A. (2009). La poesia femminile dagli anni settanta a oggi. Percorsi di analisi testuale, tesi di dottorato in cotutela, Université Paris IV Sorbonne, Università degli studi di Trieste.

\section{SITOGRAFIA}

Lo Castro, G. "Pupara sono". Il teatro bruciante della poesia di Jolanda Insana. Progetto Oblio, Osservatorio Bibliografico della Letteratura Italiana Otto-novecentescac, III, 60-68. Retrieved from http://www.progettoblio.com/files/d9-10.pdf [accesso 12.11.2017].

Turbativa d'incanto di J. Insana per SEMINARIA-Auser, con La cultura accanto, 8 maggio 2014, Cosenza. Retrieved from https://www.youtube.com/watch?v=c9-RE12QnTc [accesso 20.10.2017]. 